Dabei ist aber noch folgende wichtige Tatsache, die $B$ uxt on ermittelte, hervorzuheben, Die Lage der ersten Füllungszone hăngt, genau wie bei der gegenseitigen Einwirkung entgegengesetzt geladener Hydrosole, von den Mengenverhaltnissen des Hydrosoles und des hinzutretenden Elektrolyten ab, verschiebt sich also, wenn die Untersuchung unter denselben Verhaltnissen vorgenommen wird, regelmaBig mit Aenderung der Konzentration sowohl des Hydrosoles als des Elektrolyten. Die Lage des $z$ w e i t e $n$ Schwellenwertes des Elektrolyten dagegen ist unabhăngig von der Konzentration des Hydrosoles und nur abhanngig von der Konzentration des Elektrolyten.

Alle diese Erscheinungen lassen sich nun auch an den Jodsilberhydrosolen reproduzieren, auch sie geben unregelmäBige Reihen, deren Ursache keinem Zweifel unterliegt. Man stelle sich beispielsweise ein Hydrosol $A_{2}$ vor, welches nach dem Gesagten entstanden ist durch langsames Einfließen von $48 \mathrm{~cm} 1 / 1 \mathrm{Ag} \mathrm{NO}_{3^{-}}$ Lossung in $50 \mathrm{ccm} 1 / 20 \mathrm{KJ}$-Lösung. Fügt man zu diesem Hydrosole etwa $0,5 \mathrm{ccm}^{2 / 20} \mathrm{Ag} \mathrm{NO}_{3}$ Losung oder irgend eine andere $\mathrm{Ag} \mathrm{NO} \mathrm{S}_{\mathrm{s}}$-Lösung, die den gleichen $\mathrm{Ag} \mathrm{NO}_{3}$ - Gehalt besitzt, hinzu, so entsteht keine Pallung, man hat eine Nachzone vor sich. Bringt man aber mehr Silbernitrat, etwa eine Menge, die 1,7 ccm $1 / 20 \mathrm{Ag} \mathrm{NO}_{3}$ entspricht, in dasselbe Hydrosol, so bemerkt man unvollkommene Fällung, die vielmehr ein Optimum aufweist, wenn eine $2,0 \mathrm{~cm} 1 / 20$ norm. entsprechende Silbernitratmenge zum Hydrosole tritt, d. h. das Fallungsoptimum und die Lage der'Pallungszone ist abhangig von der absoluten Menge des hinzutretenden Elektrolyten sowohl, als des Hydrosoles oder vielmehr des in demselben enthaltenen hydrosolbildenden lons: sie müssen einander aquivalent sein. Auch eine etwas grobere Menge, z. B. $4 \mathrm{ccm}^{1 / m} \mathrm{Ag} \mathrm{NO}_{3}$ erzeugt noch Fallung, weil man noch nicht über die Fallungszone hinaus ist. Pügt man aber $\mathrm{mu}$ dem beschriebenen Hydrosole beispielsweise $10 \mathrm{ccm}$ oder eine beliebig viel größere Menge 1/m $\mathrm{Ag} \mathrm{NO}$ hinzu, so entsteht keine Fallung, vielmehr erhalt man nun ein positiv geladenes Jodsilberhydrosol (das negativ geladene wurde also umgeladen) und man befindet sich in der sekundären Nichtflockungszone. Nur eine erheblich konzentriertere als $1 / 20 \mathrm{Ag} \mathrm{NO}_{3}$-Lösung, z. B. eine 1/6 norm. erzeugt in dem negativen Hydrosole wieder Fallung, vorausgesetzt, da $B$ die in ihr enthaltene Ag NO -Menge wesentljch die $0,5 \mathrm{ccm}^{2} / 20 \mathrm{Ag} \mathrm{NO}_{3}$ entsprechende übersteigt. Diese Fallung erstreckt sich bis zu den höchsten $\mathrm{AgNO}_{3}$-Konzentrationen (abgesehen natürlich von der bald sichtbar auftretenden Bildung des loslichen Komplexsalzes) und ist nur abhangig von der Konzentration des Elektrolyten, nicht von den Verhältnissen der Elektrolyt- zur Hydrosolmenge.

Aus dern Angeführten wird man erkennen, dab die Jodsilberhydrosole hochst durchsichtige und einfache Versuchsobjekte darbieten, die imstande sind, eine ganze Reihe von Tatsachen der Kolloidfällung anschaulich vor Augen zu führen und ihrer allgemeinen Erklärung die Wege zu ebnen.

\title{
Ueber die Koagulation des Berlinerblaus.
}

\author{
Von N. Pappada. \\ (Eingegangen 6. November 1909) \\ (Aus dem chemischen Laboratorium des Technischen Instituts in Cremona.)
}

In vorliegender Mitteilung sollen die Resultate meiner Versuche über die Koagulation des Berlinerblaus mitgeteilt werden. Die Ergebnisse sind analog denen, welche ich bei der Kieselsäure ${ }^{1}$ ) erhalten und bereits veröffentlicht habe. Der Unterschied besteht nur darin, daB bei der Kieselsăure die Ausflockung erst nach einiger Zeit stattfindet und daß die Koagulationsgeschwindigkeit dort nach der Dauer des Koagulierungsvorganges gemessen wird; beim Berlinerblau geht die Ausflockung wegen der elektrolytischen

1) Societh Chimica di Ruma 1904; Gazz. Chim. Ital. 1905
Leitung auf einmal vor sich, und die Koagulationswirkung der Elektrolyte wird gemessen durch die Aenderung der Konzentration des Elektrolyten und der Menge der aktiven Masse. Zunalchst ist hervorzuheben, daB die Resultate gleich sind, wenn es sich um verschieden konzentrierte Kolloidlossungen handelt; ich habe stets die gleichen Resultate erhalten. Es scheint daher die Konzentration des Kolloides keinen nenmenswerten. Einflub auf das Koagulationsphănomen zu haben. Dies unterstützt die Ansicht, gemäB welcher die Ausflockung des Kolloides durch Ausgleich der elektrischen 
Ladungen bewirkt wird (nach meiner formulierten Annahme) ${ }^{2}$ ).

In entsprechender Weise hat das Berlinerblau im Gegensatze 7ur Kieselsäure auch nicht die Eigenschaft zu gelatinieren.

Die kolloide Lossung wurde bereitet durch Auflösen des mit Eisenchlorid und Ferrozyankalium erhaltenen Niederschlages in einer Lösung von Oxalsäure. Die filtrierte Läsung war vollkommen klar und wurde durch dreißigtägige Dialyse gereinigt, bis man in AuBenwasser keine Elektrolyten mehr nachweisen konnte.

Ich untersuchte Lösungen mit $0,2,0,4,0,5$ und 2 Proz. Gehalt an Berlinerblan. Die Flüssig. keiten lassen beim Hinzufügen von Elektrolyten das Kolloid in Flocken ballen, welche von der gleichen Farbe sind wie die Lösung und sich bald am Boden des Gefübes absetzen. In diesem Palle ist es nicht nöglich, wie bei der Kieselsäure, die Koagulierungsgeschwindigkeit nach der Dauer des Koagulierens zu verfolgen. Die Elektrolyte bewirken in der kolloiden Lösung sofortige Koagulation, und die Koagulierungstatigkeit wird hestimmt durch diese

7) Gazz. Chim. Ital. 1906.
Aenderung in der Konzentration der Elektrolyte. Ich benütze hierbei stets verdünnte Lösungen $\left(\frac{N}{10}, \frac{N}{20}, \frac{N}{50}, \frac{N}{100}\right)$, bei welchen man die Dissoziation als vollständig betrachten kann.

Versuche mit zusammengesetzten nicht dissozirten organischen Losungen.

Die $\frac{\mathrm{N}}{10}$ und $\mathrm{N}$-Lösungen, ferner auch konzentriertere von Methyl-, Aethyl-, PropylAlkohol, Aethylenglykol, Glyzerin, Mannit, Glukose, J.ävulose, Saccharose koagulieren die kolloide Lösung von Berlinerblau ticht.

\section{Versuche mit Elehtrolyten.}

Die Resultate derselben sind in den nachfolgenden Tabellen niedergelegt. Die Menge der Berlinerblaulosung beträgt $2 \mathrm{ccm}$. Die Elektrolytmengen wurden zum Teil tropfenweise hinzugefügt und es bedeuten 30 Tropfen der Lösung 1 ccm*).

*) Anm. d. Red.: Es ist zu bedauem, daB der Verf. nicht eine Umrechnung der Fallungskonzentrationen auf molekulare Konzentrationen vorgenommen hat.

Wo. 0 .

Tabelle I

Versuche aber den EInflug einwertiger Kationen auf die Koagulation.

\begin{tabular}{|c|c|c|c|c|c|c|}
\hline Koagulierende Losung & $\begin{array}{l}\text { Konzertration } \\
\text { (N = Normallosung) }\end{array}$ & \multicolumn{5}{|c|}{ Verlauf der Koagulation } \\
\hline $\mathrm{HCl}$ & $\frac{N}{10}$ & die Koagulierung & erfolgt & durch & & Tropfen \\
\hline $\mathrm{CsCl}$ & $n$ & $\Rightarrow$ & " & » & 2 & " \\
\hline $\mathrm{Rb} \mathrm{Cl}$ & » & $n$ & $n$ & $n$ & 10 & * \\
\hline $\mathrm{KCl}$ & 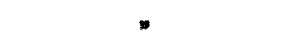 & & $*$ & - & & $\mathrm{ccm}$ \\
\hline $\mathrm{NaCl}$ & * & keine Koaguhtio & & & & \\
\hline $\mathrm{LiCl}$ & $n$ & die Koagulierun & erfolgt & durch & & \\
\hline $\mathrm{HNO}_{3}$ & $"$ & & enongt & & $\begin{array}{l}1 \\
2\end{array}$ & Tropten \\
\hline $\mathrm{CsNO}_{3}$ & $m$ & $n \quad n$ & $z$ & $"$ & $10-15$ & $"$ \\
\hline $\mathrm{RbNO}_{4}$ & $n$ & $" n$ & $"$ & $"$ & $1,5-2$ & $\mathrm{ccm}^{\prime \prime}$ \\
\hline $\begin{array}{l}\mathrm{KNO}_{3} \\
\mathrm{NaNO}_{3}\end{array}$ & " & keine Koagulatic & & $"$ & & \\
\hline $\begin{array}{l}\mathrm{LiNO}_{3} \\
\mathrm{H}_{3} \mathrm{SO}_{4}\end{array}$ & $\Rightarrow$ & die" Koagulierung & erfolgt & durch & 1 & Tropfen \\
\hline $\mathrm{Cs}_{2} \mathrm{SO}_{4}$ & $"$ & $\Rightarrow$ & * & 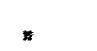 & 2 & 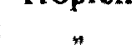 \\
\hline $\mathrm{Ro}_{2} \mathrm{SO}_{4}$ & $n$ & $*$ & $n$ & * & 10 & . \\
\hline$x_{0}, \mathrm{SO}_{4}$ & $n$ & $n \quad n \quad *$ & $m$ & . & & $.5 \mathrm{ccm}$ \\
\hline $\mathrm{Na}_{2} \mathrm{SO}_{4}$ & , & keine Koagulatic & & & & \\
\hline $\mathrm{Li}_{2} \mathrm{SO}_{4}$ & $\stackrel{n}{N}$ & $"$ & & & & \\
\hline $\mathrm{NaCl}$ & 2 & die Koagulierun & erfolgt & durch & & $\operatorname{ccm}$ \\
\hline $\mathrm{N}_{2}: \mathrm{YO}_{3}$ & x & $"$ & " & * & 2 & $"$ \\
\hline $\mathrm{Na}_{2} \mathrm{SO}_{4}$ & , & $"$ & $n$ & $n$ & 2 & 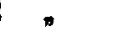 \\
\hline
\end{tabular}


Die koagulierende Kraft der dreiwertigen Kationen kann nicht auf die Gegenwart der Wasserstoffionen zurückgeführt werden, welche von der hydrolytischen Spaitung des Salzes herstammen, denn die Säuren haben keine so kraftige koagulierende Wirkung wie die Salze von Aluminium, Chrom und Eisen.

Meine Versuche über die Koagulation des Berlinerblaus führen mich zu nachstehenden Folgerungen :

1. Die Lösungen der organischen nicht dissoziierten Stoffe rufen keine Ausflockung des Berlinerblaues hervor.

2. Die Elektrolyte rufen Koagulation hervor; das Kolloid hat positive elektrische Ladung.

a) Die Koagulationswirkung durch Salze der einwertigen Kationen erfolgt, wächst mit dem Atomgewicht der Kationen, und zwar vom Lithium zum Cäsium.
ק) Die Koagulationswirkung der Salze der ein-, zwei-, dreiwertigen Kationen wächst mit der Stärke der elektrischen Ladung derselben. Zwischen der Koagulation bei der Kieselsäure und der des Berlinerblaus besteht nur ier Unterschied, dal sie im ersten Fall langsam im andern aber gleichsam plötzlich vor sich geht; der Grund dieses verschiedenen Verhaltens liegt in der Struktur der einzelnen Kolloide. Darauf werde ich in einer andern Arbeit über die Gelatinierung der Kieselsăure zutückkommen. Eirı writerer Unterschied zwischen den beiden Lösungen besteht darin, daß die Kieselsăure gelatmiert. das Berlinerblau aber nicht.

Endlich sind die Wasserstoffionen unter den einwertigen Ionen die am stärksten wirkenden Koagulierungsmittel für das Berlinerblau, während sie bei der Kieselsäure zur kolloiden Lösung derselben führen.

Tabelle II. Versuche über den EinfluB der Anionen auf die Koagulation.

\begin{tabular}{|c|c|c|}
\hline Koagulierende Losung & Konzentration & Verlauf der Koagulation \\
\hline $\begin{array}{l}\mathrm{CsJ} \\
\mathrm{CsBr} \\
\mathrm{CsCl} \\
\mathrm{CsNO}_{3} \\
\mathrm{Cs}_{2} \mathrm{SO}_{4}\end{array}$ & $\begin{array}{c}\mathrm{N} \\
10 \\
" \\
" \\
"\end{array}$ & $\begin{array}{l}\text { gleiche Geschwindigkeit der Koagulation; } \\
\text { sie wurde durch } 2 \text { Tropfen hervorgerufen. }\end{array}$ \\
\hline $\begin{array}{l}\mathrm{RbJ} \\
\mathrm{RbBr} \\
\mathrm{RbCl} \\
\mathrm{RbNO}_{3} \\
\mathrm{Rb}_{2} \mathrm{SO}_{4}\end{array}$ & $"$ & $\begin{array}{l}\text { gleiche Koagulationsgeschwindigkeit; } \\
\text { die Koagulierung erfolgt durch } 10 \cdots 15 \text { Tropten. }\end{array}$ \\
\hline $\begin{array}{l}\mathrm{KJ} \\
\mathrm{KBr} \\
\mathrm{KCl} \\
\mathrm{KNO}_{3} \\
\mathrm{~K}_{2} \mathrm{SO}_{4}\end{array}$ & $\begin{array}{l}" \\
" \\
" \\
"\end{array}$ & $\begin{array}{l}\text { gleiche Koagulierungsgeschwindigkeit; } \\
\text { die Koagulierung erfolgt durch ungefăhr } 2 \mathrm{ccm} \text {. }\end{array}$ \\
\hline $\begin{array}{l}\mathrm{NaJ} \\
\mathrm{NaBr} \\
\mathrm{NaCl} \\
\mathrm{NaNO}_{3} \\
\mathrm{Na}_{2} \mathrm{SO}_{4}\end{array}$ & $\begin{array}{l}* \\
" \\
"\end{array}$ & keine Koagulation bei Anwendung von $3 \mathrm{ccm}$. \\
\hline $\begin{array}{l}\mathrm{LiCl} \\
\mathrm{LiNO}_{3} \\
\mathrm{Li}_{2} \mathrm{SO}_{4}\end{array}$ & $:$ & keine Kodgulation durch 3 und $4 \mathrm{ccm}$. \\
\hline
\end{tabular}


Tabelle [I]

Versuche über den Einflus zweiwertiger Kationen auf die Koagulation.

\begin{tabular}{|c|c|}
\hline Koagullerende Losung & Konzentration \\
\hline & $\mathbf{N}$ \\
\hline $\mathrm{BaCl}_{2}$ & 10 \\
\hline & $\mathbf{N}$ \\
\hline $\mathrm{BaJ}_{2}$ & 10 \\
\hline & $\mathbf{N}$ \\
\hline $\mathrm{Ba}\left(\mathrm{NO}_{\mathrm{g}} \mathrm{k}\right.$ & 10 \\
\hline $\mathrm{SrCl}_{\mathrm{B}}$ & $\mathbf{N}$ \\
\hline $\mathrm{SrCl}_{2}$ & 10 \\
\hline $\mathrm{CaCl}$ & $\mathbf{N}$ \\
\hline $\mathrm{CaCb}_{2}$ & 10 \\
\hline $\mathrm{BnOl}_{n}$ & $\mathbf{N}$ \\
\hline $\mathrm{baCl}_{\mathrm{g}}$ & 20 \\
\hline & $\mathbf{N}$ \\
\hline $\mathrm{SrCl}_{2}$ & 20 \\
\hline & $\mathbf{N}$ \\
\hline $\mathrm{CaCh}_{2}$ & 20 \\
\hline $\mathrm{CdCl}_{2}$ & $\mathbf{N}$ \\
\hline $\mathrm{CaCl}_{2}$ & 10 \\
\hline & $\mathbf{N}$ \\
\hline$\bullet$ & 20 \\
\hline & $\mathbf{N}$ \\
\hline$"$ & 50 \\
\hline 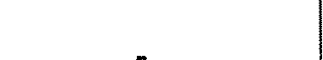 & $\mathbf{N}$ \\
\hline$n$ & 100 \\
\hline $\mathrm{CdSO}_{4}$ & $\frac{N}{100}$ \\
\hline
\end{tabular}
Verlauf der Koagulation

koagulieren gleich stark; die Koagulation wird hervorgerufen durch 1 Tropfen.

ruft die Koagulierung mit 2 Tropfen hervor; mit einem Tropfen sehr langsam.

die Koagulation erfolgt bei 2 Tropfen, bei einem tritt keine Koagulierung ein.

Koagulation bei 1 Tropfen.

1,2 und 3 Tropfen rufen keine Koagulation hervor.

wie beim Strontium $\frac{N}{20}$

koaguliert hei 1 Tropfen.

koaguliert bei 3 Tropfen.

Tabelle IV

Versuche über den Einflub dreiwertiger Kationen auf die Koagulation

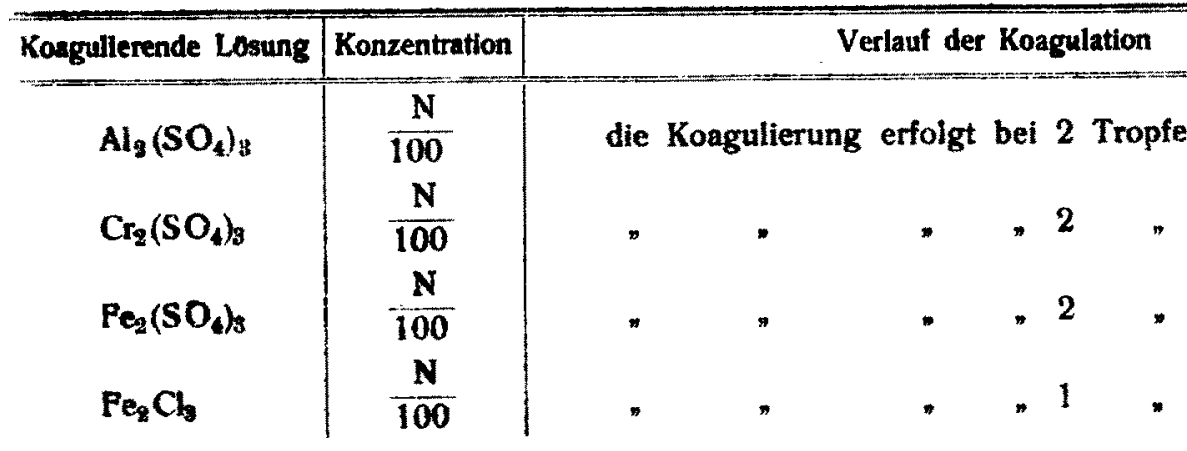

NB. Ein Auszug aus dieser Arbeit ist veröffentlicht in der "Gazz. Chim. Ital." (Sulla natura della coagulatione). 